\title{
Valorisation nutritive d'un grignon d'olive traité à la soude. Utilisation digestive des constituants des parois cellulaires
}

\author{
Eduarda MOLINA et J.F. AGUILERA \\ avec la collaboration technique de Encarnación COLMENERO \\ Estación Experimental del Zaidín (C.S.I.C.) \\ Profesor Albareda, 1. 18008 Granada, Espagne
}

\begin{abstract}
Résumé
Afin de connaître l'utilisation digestive et la contribution des constituants des parois cellulaires à la valeur nutritive d'un grignon d'olive épuisé tamisé et traité avec $0,5,7,5$ et $10 \mathrm{~g} \mathrm{de}$ $\mathrm{NaOH} / 100 \mathrm{~g}$ de sous-produit, on a estimé leur digestibilité in vivo chez des moutons. Dans une publication précédente (Agullera \& MoLina, 1986) nous avons décrit l'effet du traitement à la soude sur la valeur nutritive du grignon d'olive et nous avons aussi étudié la précision de quelques méthodes de laboratoire pour estimer la valeur nutritive de ce sous-produit.

Le traitement à la soude du grignon d'olive augmente significativement $(\mathrm{P}<0.001)$ la digestibilité de NDF, ADF, ADL et cellulose. Par contre, la digestibilité de l'hémicellulose n'est pas modifiée. Cela explique l'augmentation de la valeur énergétique du grignon d'olive déjà observée (Aguilera \& Molina, 1986).

L'absorbance à $280 \mathrm{~nm}$ des fractions solubles après extraction du grignon traité à la soude avec l'eau distillée est significativement $(\mathrm{P}<0.001)$ corrélée avec la digestibilité et les contenus digestibles de la matière organique et les différents composants pariétaux et elle permet de prédire avec précision ces paramètres. Cette méthode donne des écarts-types résiduels inférieurs à ceux obtenus avec les méthodes chimiques et biologiques de prévision (Aguilera \& Molina, 1986).

En conclusion, l'effet du traitement à la soude du grignon d'olive sur la digestibilité des constituants pariétaux est important. Malgré cela, la digestibilité reste faible, ce qui explique la faible valeur nutritive du grignon d'olive dont la plus grande partie de la matière organique est représentée par les constituants pariétaux.
\end{abstract}

Mots clés: Grignon d'olive, traitement à la soude, digestibilité, parois cellulaires.

\section{Introduction}

Les grignons d'olive peuvent représenter une source d'aliment d'une certaine importance dans des régions traditionnellement caractérisées par leur déficit en fourrages s'ils sont traités de façon appropriée pour accroître leur valeur nutritive.

Dans une publication précédente (Aguilera \& Molina, 1986) nous avons observé l'effet bénéfique du traitement à la soude d'un grignon d'olive sur sa valeur énergétique. Etant donné que le grignon d'olive est surtout une source de composants de la 
paroi cellulaire (la plupart de son azote - 75 p. 100 - est attachée aux parois) il est de grand intérêt de connaître la digestibilité de ces constituants. Les données sur l'utilisation digestive des composants de la paroi cellulaire des grignons d'olive sont très rares. De plus, il est bien démontré que les différentes espèces végétales répondent d'une façon différente aux traitements alcalins (CHANDRA \& JACKSON, 1971 ; ReXeN \& Vestergaard Thomsen, 1976 ; Chandra et al., 1985).

Ainsi, avons-nous cherché à évaluer la digestibilité des différents constituants des parois cellulaires d'un grignon d'olive traité à la soude d'après une série d'essais menés sur des moutons (Aguilera \& Molina, 1986) avec l'objectif de connaître quelle est la contribution de chacun de ces constituants à l'amélioration de la valeur énergétique entraînée par le traitement à la soude.

\section{Matériel et méthodes}

L'étude a porté sur les échantillons d'aliments et de fèces issus d'essais précédents dont l'objectif était de connaître l'effet du traitement à la soude du grignon d'olive sur sa digestibilité in vivo chez des moutons (Aguilera \& Molina, 1986) : un grignon d'olive épuisé et partiellement tamisé fut traité par pulvérisation avec $0,5,7,5$ et $10 \mathrm{~g}$ de $\mathrm{NaOH} / 100 \mathrm{~g}$ de grignon. Les moutons recevaient quatre régimes témoins constitués de $400 \mathrm{~g}$ de foin de luzerne, $500 \mathrm{~g}$ de concentré (orge + tourteau de tournesol) et $100 \mathrm{~g}$ de grignon non traité ou traité à la soude, et quatre régimes expérimentaux formulés en remplaçant $200 \mathrm{~g}$ de la quantité du régime témoin offerte aux animaux avec la dose de soude correspondante. Avec la présence du grignon dans les régimes témoins, nous essayions d'éviter des phénomènes associatifs qui pourraient apparaître avec l'incorporation du grignon, dans les régimes expérimentaux. Chacun des régimes étudiés a été offert à 6 moutons. La digestibilité des grignons d'olive a été calculée par différence.

Pour le dosage de la fraction pariétale (NDF), de la fraction lignocellulosique (ADF) et de la lignine (ADL) nous avons suivi les techniques décrites par Goeking \& VAN Soest (1970). On a utilisé des échantillons d'aliments et de fèces. Préalablement au dosage des composants, les fèces ont été séchées à $80^{\circ} \mathrm{C}$, équilibrées à température ambiante pendant 48 heures et moulues $(1 \mathrm{~mm})$; elles ont été conservées à $-25^{\circ} \mathrm{C}$.

Pour le dosage de l'absorbance à $280 \mathrm{~nm}$, nous avons suivi la technique développée par Nefzaoui (1985), en tenant compte du fait que les fractions solubles aux détergents neutre et acide de Van Soest présentent constamment un maximum d'absorbance à $280 \mathrm{~nm}: 1 \mathrm{~g}$ de grignon moulu $(1 \mathrm{~mm})$ est soumis à ébullition, sous reflux, dans de l'eau distillée pendant 1 heure, filtré sur papier et amené au volume de $100 \mathrm{ml}$. Après dilution 1 : 100 l'absorbance à $280 \mathrm{~nm}$ est mesurée au spectrophotomètre.

\section{Analyses statistiques}

Les résultats expérimentaux ont été soumis à une analyse de variance et les différences entre traitements ont été testées par la méthode de la plus petite différence significative de Duncan. 


\section{Résultats}

La composition en constituants de la paroi cellulaire des régimes étudiés est présentée dans la tableau 1. Elle reflète l'effet du traitement à la soude sur les constituants pariétaux du grignon d'olive qui a été décrit dans la publication précédente (Aguilera \& Molina, 1986).

\section{TABleaU 1}

Composition en constituants des parois cellulaires des régimes étudiés ( $\mathrm{g} / \mathrm{Kg} \mathrm{MS}$ ). Composition of cell wall components of control and experimental diets $(\mathrm{g} / \mathrm{Kg} \mathrm{DM})$.

\begin{tabular}{|c|c|c|c|c|c|c|c|c|}
\hline Régimes (1) & $\mathrm{RT}_{0}$ & $\mathrm{RT}_{5}$ & $\mathrm{RT}_{7.5}$ & $\mathrm{RT}_{10}$ & $\mathrm{RE}_{0}$ & $\mathrm{RE}_{5}$ & $\mathrm{RE}_{7.5}$ & $\mathrm{RE}_{10}$ \\
\hline$\ldots \ldots \ldots$ & 353 & 360 & 354 & 342 & 478 & 466 & 443 & 393 \\
\hline ADF & 257 & 269 & 263 & 258 & 353 & 384 & 362 & 340 \\
\hline ADL $\ldots \ldots \ldots \ldots$ & 92 & 98 & 95 & 92 & 168 & 177 & 165 & 151 \\
\hline Hemicellulose $\ldots \ldots \ldots$ & 96 & 91 & 91 & 84 & 101 & 79 & 78 & 50 \\
\hline Cellulose . . . . . . . . . & 165 & 180 & 168 & 167 & 185 & 206 & 196 & 189 \\
\hline
\end{tabular}

(1) $\mathrm{RT}=$ Régime témoin $; \mathrm{RE}=$ Régime expérimental. Le suffixe indique le pourcentage de soude dans le grignon d'olive de la ration.

$R T$ Control diet; $R E=$ Experimental diet. Subscripts indicate the $\mathrm{NaOH}$ percentage in the dietary cake.

TABleau 2

Coefficients de digestibilité moyens des constituants des parois cellulaires du grignon d'olive épuisé tamisé $(n=6)$. Effet du traitement à la soude (p. 100).

Cell wall components digestibility for olive cake $(n=6)$. Effect of soda treatment (p. 100).

\begin{tabular}{|c|c|c|c|c|c|c|}
\hline \multirow{2}{*}{$\begin{array}{l}\text { Digestibilités } \\
\text { Digestibilities }\end{array}$} & \multicolumn{4}{|c|}{$\begin{array}{c}\mathrm{g} \mathrm{NaOH} / 100 \mathrm{~g} \text { de grignon } \\
\mathrm{g} \mathrm{NaOH} / 100 \mathrm{~g} \text { olive cake }\end{array}$} & \multirow{2}{*}{$\begin{array}{l}\text { ESM } \\
S E M\end{array}$} & \multirow{2}{*}{$\begin{array}{l}\text { Niveau } \\
\text { de signi- } \\
\text { fication } \\
\text { Level of } \\
\text { signi- } \\
\text { ficance }\end{array}$} \\
\hline & 0 & 5 & 7,5 & 10 & & \\
\hline$\ldots \ldots \ldots \ldots$ & $15,5^{\mathrm{a}}$ & $34,2^{b}$ & $32,2^{h}$ & $31,4^{\mathrm{h}}$ & 2,00 & $* * *$ \\
\hline ADF & $8,6^{\text {a }}$ & $27,8^{b}$ & $25,1^{\mathrm{h}}$ & $27,9^{\mathrm{h}}$ & 2,58 & $* * *$ \\
\hline ADL $\ldots \ldots \ldots \ldots$ & 14,2 & $27.9^{b}$ & $24,7^{b}$ & $26,2^{h}$ & 1,90 & $* * *$ \\
\hline$C C \ldots \ldots \ldots$ & $35,6^{\prime \prime}$ & $45,1^{\circ}$ & $46,1^{n}$ & $50,5^{\mathrm{h}}$ & 3.83 & $*$ \\
\hline Hemicellulose & 52,8 & 70.1 & 79,0 & 69,2 & 10,54 & NS \\
\hline Cellulose ...... & 2,9 " & $27,7^{\mathrm{h}}$ & $26,6^{\mathrm{h}}$ & $31,9^{\mathrm{h}}$ & 4,32 & $* * *$ \\
\hline
\end{tabular}

NS $=\mathrm{P}>0.05:{ }^{*}=\mathrm{P}<0.05:{ }^{* * *}=\mathrm{P}<0,001$.

Les moyennes dans une même ligne, suivies de lettres différentes sent significativement différentes $(P<0.05)$. (Test de Duncan)

Within a row. means bearing unlike superscripts are significanly different $(P<0.05)$ (Duncan Tesn). 
Nous avions observé que le contenu en parois cellulaires du grignon d'olive diminue significativement $(68,1$ à $58,6 \%)$ avec le traitement à la soude. Cette diminution était due à la solubilisation des hémicelluloses $(10,8$ à $2,7 \%)$. Par contre, les fractions $\mathrm{ADF}$ et $\mathrm{ADL}$ ne se modifient pas $(53,7$ à $56,0 \%$ et 29,7 à $28,4 \%$, respectivement). Les résultats furent exprimés par rapport à la matière organique afin d'éviter l'effet de la variation du contenu en cendres due à l'addition de la soude.

Les résultats de digestibilité des constituants de la paroi cellulaire du grignon d'olive soumis aux différents traitements sont donnés au tableau 2. La digestibilité des fractions NDF, ADF, ADL et cellulose augmente d'une façon remarquable et significative $(P<0.001)$ après le traitement à la soude. Par contre, l'augmentation de la digestibilité des hémicelluloses du grignon comme conséquence du traitement à la soude, n'est pas significative. Nos résultats montrent que l'intensité de l'effet avantageux du traitement à la soude sur la digestibilité diminue avec les doses de soude plus élevées. La digestibilité de la fraction des contenus cellulaires est améliorée $(P<0.05)$ par le traitement à la soude, mais il n'y a pas de différences significatives entre les trois doses d'addition de l'alcali.

La soude agit sur le grignon d'olive en libérant des groupes phénoliques qui présentent leur maximum d'absorbance à $280 \mathrm{~nm}$ (Fig. 1). La mesure de l'absorbance à cette longueur d'onde peut s'utiliser comme un estimateur de la digestibilité des constituants de la paroi cellulaire. De plus, étant donné que dans le cas du grignon d'olive les constituants pariétaux représentent la plus grande partie de la matière organique, la mesure de l'absorbance peut servir aussi à estimer la digestibilité de la matière organique. Les équations de régression simples, obtenues à partir des données de digestibilité des composants de la paroi cellulaire et des valeurs d'absorbance à $280 \mathrm{~nm}$, sont présentées dans la tableau 3.

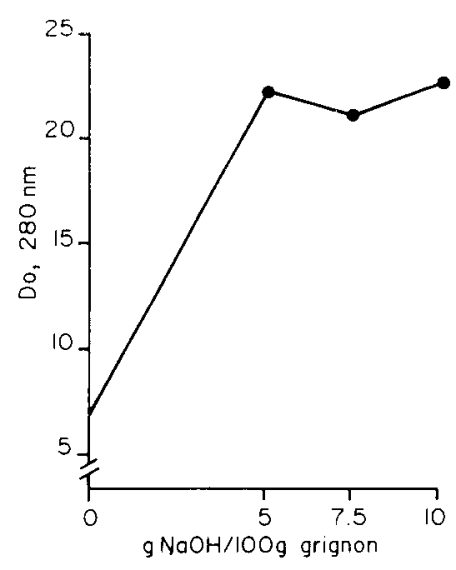

FIG. 1

Densité optique à $280 \mathrm{~nm}$ du grignon d'olive traité avec différentes concentrations de soude. Optical density at $280 \mathrm{~nm}$ of a screened solvent extracted olive cake sprayed with different solutions of sodium hydroxide. 


\section{TABLEAU 3}

Régressions simples entre la digestibilité in vivo ou les contenus digestibles de différents constituants du grignon d'olive traité à la soude et lu densité optique à $280 \mathrm{~nm}$ de la fraction soluble après extraction à l'eau distillée.

Linear regressions of in vivo digestibility or digestible contents of some constituents of a screened solvent extracted olive cake treated with soda and optical density at $280 \mathrm{~nm}$ of the fraction extracted with distilled water.

\begin{tabular}{|c|c|c|c|c|c|c|}
\hline$y=$ & $a+$ & bx & $\mathrm{r}$ & RSD & $\mathrm{n}$ & $\begin{array}{c}\text { Niveau } \\
\text { de signi- } \\
\text { fication } \\
\text { Level of } \\
\text { signi- } \\
\text { ficance }\end{array}$ \\
\hline DNDF in vivo, $\%$ & 7,96 & $+1,10$ Do & 0,990 & 1,510 & 8 & $* * *$ \\
\hline DADF in vivo, $\% \ldots$ & 0,286 & $+1,19$ Do & 1,000 & 0,353 & 8 & $* * *$ \\
\hline DADL in vivo, $\% \ldots$ & 8,62 & $+0,787 \mathrm{Do}$ & 0,994 & 0,841 & 8 & $* * *$ \\
\hline DMO in vivo, $\% \ldots$ & 23,01 & $+0,551 \mathrm{Do}$ & 0,994 & 0,591 & 8 & $* * *$ \\
\hline MEB,$\% \quad \ldots \ldots$ & 15,28 & $+0,679$ Do & 0,925 & 2,650 & 8 & $* * *$ \\
\hline NDFD, g/kg MO ... & 62.19 & $+6,33$ Do & 0,947 & 20,580 & 8 & $* * *$ \\
\hline ADFD, $\mathrm{g} / \mathrm{kg} \mathrm{MO} \ldots$ & 2,056 & $+6,75$ Do & 1,000 & 1,239 & 8 & $* * *$ \\
\hline ADLD, $\mathrm{g} / \mathrm{kg} \mathrm{MO}$ & 27,04 & $+2,13$ Do & 0,991 & 2,817 & 8 & $* * *$ \\
\hline MOD, g/kg MS ... & 225,51 & $+3,17$ Do & 0,979 & 6,339 & 8 & $* * *$ \\
\hline $\mathrm{EM}, \mathrm{MJ} / \mathrm{kg}$ MS $\ldots$ & 3,42 & $+0,119 \mathrm{Do}$ & 0,944 & 0,395 & 8 & $* * *$ \\
\hline
\end{tabular}

***: $P<0,001$

DNDF in vivo : Digestibilité des parois cellulaires - Cell wall digestibility.

DADF in vivo : Digestibilité des lignocelluloses - Lignocellulose digestibility.

DADL in vivo: Digestibilité de la lignine - Lignin digestibility.

DMO in vivo: Digestibilité de la matière organique - Organic matter digestibility.

MEB : Energie métabolisable/Energie brute - Metabolizable energy/Gross energy.

NDFD: Neutre détergent fibre digestible - Digestible neutral detergent fibre.

ADFD : Acide détergent fibre digestible - Digestible acid detergent fibre.

ADLD : Acide détergent lignine digestible - Digestible lignin.

MOD : Matière organique digestible - Digestible organic matter.

EM : Energie métabolisable - Metabolizable energy.

\section{Discussion-conclusions}

L'augmentation de la digestibilité des constituants de la paroi cellulaire (NDF) du grignon, suite au traitement à la soude, que nous avons observée dans nos essais peut être attribuée tant à la rupture des ponts d'hydrogènes (WhistLer \& TENG, 1970 ; BACON, 1979) qu'à la saponification des liaisons ester entre la cellulose et l'hémicellulose (Feist et al., 1970). Cela peut favoriser la perte de la structure des parois cellulaires et par conséquent, l'accès des microorganismes du rumen au complexe lignocellulosique en améliorant la digestibilité de la cellulose. La libération de groupes phenyl et acétyl pourrait expliquer la faible et non significative amélioration de la digestibilité des hémicelluloses. 
La digestibilité de la fraction ADL varie entre 14,2 et $26,2 \%$. HarTLEY (1973) et GaIllard \& RichaRdS (1975) ont montré que l'activité des microorganismes du rumen en présence des hydrates de carbone non structuraux peut conduire à la formation de complexes solubles lignine-polyolosides, contribuant à dissoudre une fraction de la lignine ingérée qui n'est pas dosée comme lignine dans les fécès. Cela pourrait expliquer la digestibilité partielle de la lignine déjà observée par d'autres auteurs (Allison \& Osbourn, 1970 ; Minson, 1971 ; Grant et al., 1974). Mais il ne faut pas oublier que d'autres causes, basées sur les limites de la méthode analytique, peuvent expliquer la digestibilité positive de la lignine. Muntıfering (1982) signale que la destruction partielle de la lignine fécale dans le processus analytique et les différences de la nature physique et/ou chimique de la lignine dans l'aliment et les fécès sont des causes probables de la digestibilité partielle de la lignine. VAN SOEST (1982) attribue cette digestibilité à la perte possible des particules fécales les plus petites et très riches en lignine pendant le processus de filtration inclus dans la méthode analytique.

L'amélioration de la digestibilité de la matière organique et, par conséquent, de la valeur énergétique du grignon d'olive à la soude (Agullera \& MolinA, 1986) est due à l'augmentation de la digestibilité des constituants des parois cellulaires, causée aussi bien par leur solubilisation partielle que par la plus grande sensibilité du matériel non solubilisé à la dégradation microbienne. De plus, l'augmentation de la digestibilité des contenus cellulaires, fraction dans laquelle se retrouvent les hémicelluloses solubilisées après le traitement à la soude est aussi un facteur qui contribue à améliorer la digestibilité de la matière organique. Les coefficients de digestibilité apparente de la matière organique du grignon traité avec $0,5,7,5$ et $10 \mathrm{~g}$ de $\mathrm{NaOH} / 100 \mathrm{~g}$ de sousproduit furent de 26,$9 ; 35,6 ; 34,2$ et $36,3 \%$ respectivement.

L'augmentation de la vitesse de passage à travers le tractus digestif, due à une ingestion plus élevée d'eau, suite à la consommation de sodium (BoLduAN et al., 1974 ; MCManus et al., 1976 ; Berger et al., 1980) peut expliquer que des doses de $\mathrm{NaOH}$ supérieures à $5 \%$ aient un effet additionnel faible sur l'amélioration de la digestibilité des composants pariétaux. De plus, la présence de quantités élevées de sodium dans le rumen provoque une augmentation de la pression osmotique qui agit en réduisant l'activité des microorganismes cellulolytiques (BERGEN, 1970 ; Ololade et al., 1972).

Même si la comparaison des données est difficile, compte tenu de l'hétérogénéité de la composition chimique des grignons d'olive de par leur origine et le traitement technologique suivi pour l'obtention de l'huile, la digestibilité des constituants de la paroi cellulaire et la faible amélioration suite au traitement à la soude sont du même ordre que ceux mentionnés par d'autres auteurs (Nefzaoui, 1985 ; Nefzaoui \& VaNBelle, 1986). Nefzaoui (1985) a mesuré sur des ovins, la digestibilité des constituants des parois cellulaires d'un grignon d'olive épuisé tamisé et traité ou non avec $4 \%$ de soude et additionné avec $8 \%$ de mélasses et $1,5 \%$ d'urée. Les digestibilités trouvées pour les fractions NDF, ADF, ADL, hémicellulose et cellulose du grignon non traité ou traité à la soude étaient respectivement, 24,4 et $32,5 \% ; 18,1$ et $25,7 \% ; 13,6$ et $23,1 \% ; 49,4$ et $61,8 \% ; 26,0$ et $28,8 \%$.

Avec d'autres matériaux très lignifiés, différents auteurs (OrSKov, 1977 ; MichaletDoreau, 1981 ; Preston et al., 1981) ont remarqué l'importance du niveau de participation de l'aliment dans le régime total, du type d'aliments associés, du niveau d'alimentation de l'animal et, aussi de la méthode de calcul ou d'estimation de la digestibilité du matériel lignocellulosique sur les valeurs de digestibilité obtenues. 
Il a été montré que la réponse au traitement à la soude varie beaucoup selon la nature du matériel (JACKSON, 1977), probablement à cause des différences dans les proportions relatives des constituants des parois cellulaires, ainsi que des rapports physiques et chimiques entre ces constituants. Il a été suggéré que les augmentations les plus importantes de la digestibilité, suite au traitement alcalin, se trouvent pour les matériaux qui éprouvent les diminutions les plus importantes du contenu en hémicelluloses et des diminutions faibles ou aucun changement du contenu en ADL. Les études ayant porté dans notre laboratoire sur la bagasse de canne à sucre, un sous-produit qui a un degré de lignification moyen (Molina et al., 1983), ont montré que le traitement à la soude fait diminuer de façon remarquable le contenu en NDF, dû surtout à la solubilisation des hémicelluloses, et améliore remarquablement et significativement la digestibilité de NDF, ADF, ADL, hémicellulose et CC. Les résultats obtenus par Michalet-Doreau \& Demarquilly (1980) pour des coques de tournesol, Horton et al. (1982), Sehgal \& Pung (1983), Kerley et al. (1985) pour des pailles de blé, Chandra et al. (1985) pour des coques d'arachide confirment l'hypothèse précédente.

Le contenu en hémicelluloses du grignon d'olive est faite par rapport à ceux des pailles de céréale et de la bagasse de canne à sucre. Il est donc probable que l'effet du traitement à la soude sur le grignon d'olive soit moins important à cause de cela.

Les équations de régression rapportées dans le tableau 3 permettent de connaître avec une grande précision la digestibilité apparente des différents composants du grignon à partir des données d'absorbance à $280 \mathrm{~nm}$. La grande simplicité de cette technique de laboratoire la rend très intéressante pour la prévision de la digestibilité des matériaux lignocellulosiques traités à la soude, même avec des avantages par rapport aux régressions basées sur les données obtenues avec d'autres méthodes de laboratoire, aussi bien chimiques que biologiques. De fait, les écarts-types résiduels (RSD) obtenus avec la méthode d'absorbance sont inférieurs à ceux obtenus dans la publication précédente (Aguilera \& Molina, 1986) avec la méthode chimique (schéma de fractionnement de Van Soest), la méthode enzymatique et la méthode de digestibilité in vitro avec du jus de rumen.

On peut conclure que même si l'effet du traitement à la soude sur la digestibilité des constituants pariétaux est important, la digestibilité reste faible, ce qui explique la faible valeur nutritive de ce sous-produit dont la plus grande partie de la matière organique est représentée par les constituants des parois cellulaires.

Reçu en mars 1988.

Accepté en juillet 1988.

\section{Remerciements}

Nous voudrions présenter nos sincères remerciements à M. LARA qui a collaboré au traitement des analyses statistiques et à $M^{\text {llc }}$ Garcia SALCEDo qui a collaboré aux analyses chimiques. 


\title{
Summary \\ Nutritive value of a soda-treated olive cake. Digestibility of cell wall components
}

\begin{abstract}
The effect of sodium hydroxide treatment on the digestibility of cell wall constituents of a screened, solvent-extracted olive cake was assessed in the adult sheep. Solutions were sprayed to reach $0,5,7.5$ and $10 \mathrm{~g} \mathrm{NaOH} / 100 \mathrm{~g}$ by-product. The effect of sodium hydroxide treatment on the nutritive value of olive cake and the accuracy of some chemical and biological laboratory methods for estimating the nutritive value of this by-product have been studied in a previous paper (Aguilera \& Molina, 1986). Soda treatment significantly improved $(P<0.001)$ NDF, ADF, ADL and cellulose apparent digestibilities (Table 2). On the contrary, it did not increase significantly the digestibility of the hemicellulose fraction. This effect on cell wall digestibility accounts for the increase in the nutritive value of the olive cake following the $\mathrm{NaOH}$ treatment (Agullera \& Molina, 1986).
\end{abstract}

Absorbance at $280 \mathrm{~nm}$ of solutions obtained by extraction with distilled water of the olive cake after the soda treatment (Graphic 1) proved to be an excellent laboratory method to predict both the digestibility of cell wall components and the digestibility of the organic matter and the energy value of the by-product (Table 3). RSD values from this method are lower than tose derived from chemical and biological laboratory methods (Aguilera \& Molina, 1986).

In conclusion, the improvement produced by the soda treatment on the digestibility of cell wall constituents is important. However, the treated by-product has still a poor energy value as the availability of the cell wall components remains rather low and this fraction accounts for most part of the organic matter in the olive cake.

Key words: Olive cake, NaOH treatment, digestibility, cell wall.

\section{Références bibliographiques}

Aguilera J.F., Molina E., 1986. Valorisation nutritive d'un grignon d'olive traité à la soude. Ann. Zootech., 35, 205-218.

Alluson D.W., Osbourn D.F., 1970. The cellulose-lignin complex in forages and its relationship to forage nutritive value. J. Agric. Sci., 74, 23-36.

Bacon J.S.D., 1979. Plant cell wall digestibility and chemical structure. Annu. Rep. Rowett Res. Inst., 35, 99-108.

Bergen W.G., 1970. Osmolality and rumen function in sheep. J. Anim. Sci., 31, 236 (Abstr.).

Berger L.L., Klopfenstein T.J., Britton R.A., 1980. Effect of sodium hydroxide treatment on rate of passage and rate of ruminal fiber digestion. J. Anim Sci., 50, 745-749.

Bolduan G., Voigt J., Piatkowski B., 1974. Untersuchungen zum Aufchluss von Getreidestroh. 3. Einfluss der Behandlung mit Natronlange auf die Pansenfermentation in Versuchen and Kuhen. Arch. Tierernaerh., 24, 149-157.

Chandra S., Jackson M.G., 1971. A study of various chemical treatments to remove lignin from coarse roughages and increase their digestibility, J. Agric. Sci., 77, 11-17.

Chandra S., Prasad D.A., Krishna N., 1985. Effect of sodium hydroxide treatment and/or extrusion cooking on the nuritive value of peanut hulls. Anim. Feed Sci. Technol., 12, 187. 194.

Feist W.C., Baker A.J., Tarkow H., 1970. Alkali requirements for improving the digestibility of hard woods by rumen micro-organisms. J. Anim. Sci., 30, 832-835.

Gaillard B.D.E., Richards G.N., 1975. Presence of soluble lignin-carbohydrate complexes in the bovine rumen. Carbohydr. Res., 42, 135-145. 
Goering H.K., VAZN Soest P.J., 1970. Forage fiber analyses. (Apparatus, Reagents, Procedure and some Applications). Agriculture Handbook $\mathrm{n}^{\circ} 379$. Agriculture Research Service. United States Department of Agriculture, 1-20.

Grant R.J., Van Soest P.J., McDowell R.E., Perez C.B. Jr., 1974. Intake, digestibility and metabolic loss of napier grass by cattle and buffaloes when fed wilted, chopped and whole. $J$. Anim. Sci., 39, 423-434.

Hartley R.D., 1973. Lignin-carbohydrate linkages in plant cell walls. I. Carbohydrate esters of ferulic acid as components of cell walls of Lolium multiflorum. Phytoch., 12, 661-665.

Horton G.M.J., Nicholson H.H., Christensen D.A., 1982. Ammonia and sodium hydroxide treatment of wheat straw in diets for fattening steers. Anim. Feed Sci. Technol., 7, 1-10.

JACKSON M.G., 1977. Review article: The alkali treatment of straws. Anim. Feed Sci. Technol., 2, $105-130$.

Kerley M.S., Fahey Jr. G.C., Berger L.L., Gould Michael J., Barker Lee F., 1985. Alkaline hydrogen peroxide treatment unlocks energy agricultural by-products. Science, 230, 820-822.

McManus W.R., Choung C.C., Robinson V.N.E., 1976. Studies on forage cell walls. Flow and degradation of alkaliptreated rice hull digesta in the ruminant digestive tract. J. Agric. Sci., 87, $471-483$.

Michalet-Doreau B., 1981. Etude méthodologique concernant la détermination de la valeur énergétique et azotée des sous-produits. Second consultation Meeting. FAO, Santerem, Portugal. $15 \mathrm{pp}$.

Michalet-Doreau B., Demarquilly C., 1980. Valeur alimentaire des pellicules de différentes graines oléagineuses. Bull. Tech. CRZV de Theix, 39, 15-22.

Minson D.J., 1971. The influence of lignin and silicon dioxide on a summative system for asessing the organic matter digestibility of panicum. Aust. J. Agric. Res., 22, 589-598.

Molina E., BozA J., Aguilera J.F., 1983. Nutritive value for ruminants of sugar cane bagasse ensiled after spray treatment with different levels of $\mathrm{NaOH}$. Anim. Feed Sci. Technol., 9, 1-17.

Muntifering R.B., 1982. Evaluation of various lignin essays for determining ruminal digestion of roughages by lambs. J. Anim. Sci., 55, 432-438.

Nefzaoui A., 1985. Valorisation des résidus lignocellulosiques dans l'alimentation des ruminants par les traitements aux alcalis. Application aux grignons d'olive. Thèse Docteur Ing. Faculté des Sciences Agronomiques. Université Catholique de Louvain (Belgique), 345 p.

Nefzaoui A., Vanbelle M., 1986. Effects of feeding alkali-treated olive cake on intake, digestibility and rumen liquor parameters. Anim. Feed Sci. Technol., 14, 139-149.

Ololade B.G., Mowat D.N., Yao Y.T., Smith G.C., 1972. Sodium hydroxide-treated diet and body fluids parameters. J. Anim. Sci., 35, 232 (Abstr.).

Orskov E.R., 1977. Nutritional principles and evaluation of by-products, waste products and new feeds for ruminants. Livest. Prod. Sci., 4, 165-175.

Preston T.R., Parra R., Escobar A., Dixon R., 1981. Inefficient use of ruminal digestion on end-products for productive purpose on diets composed largely of alkali treated crop residues and molasses. Precirculated paper. Proc. IAED/FAO Meeting. Nov.-Dec. Vienna.

Rexen F., Vestergand Thomsen K., 1976. The effect on digestibility of a new technique for alkali treatment of straw. Anim. Feed Sci., Technol., 1, 73-83.

Sehgal J.P., PunJ M.L., 1983. Utilization of alkali-treated and neutralized wheat straw-based rations for growing goat kids. Anim. Feed Sci. Technol., 9, 155-168.

VAn Soest P.J., 1982. Nutritional Ecology of the Ruminant (Ed. Van Soest, P.J.) O \& Books, Inc., Oregon, 374 p.

Whistler R.L., Teng J., 1970. Cellulose chemistry. In : Handbook of Pulp and Paper Technology, $13-23$; 2nd eds ; K.W. Britt (Ed.). Van Nostrand Reinhold, New York. 